\title{
The Device Centric Communication System for 5G Networks
}

\author{
S K Biswash and D N K Jayakody \\ National Research Tomsk Polytechnic University, Tomsk 634050, Russia. \\ Email: skbismu@gmail.com
}

\begin{abstract}
The Fifth Generation Communication (5G) networks have several functional features such as: Massive Multiple Input and Multiple Output (MIMO), Device centric data and voice support, Smarter-device communications, etc. The objective for $5 \mathrm{G}$ networks is to gain the 1000x more throughput, 10x spectral efficiency, $100 \mathrm{x}$ more energy efficiency than existing technologies. The 5G system will provide the balance between the Quality of Experience (QoE) and the Quality of Service (QoS), without compromising the user benefit. The data rate has been the key metric for wireless QoS; QoE deals with the delay and throughput. In order to realize a balance between the QoS and QoE, we propose a cellular Device centric communication methodology for the overlapping network coverage area in the $5 \mathrm{G}$ communication system. The multiple beacon signals mobile tower refers to an overlapping network area, and a user must be forwarded to the next location area. To resolve this issue, we suggest the user centric methodology (without Base Station interface) to handover the device in the next area, until the users finalize the communication. The proposed method will reduce the signalling cost and overheads for the communication.
\end{abstract}

\section{Introduction}

The modern cellular network, using the Fourth Generation (4G), Long Term Evolution (LTE) technologies, and users are facing huge demand from users to improve its functional capacity. It seems that $4 \mathrm{G}$ has just begun to settle, the attention is now slowly turning towards future $5 \mathrm{G}$ technologies. A key feature of $4 \mathrm{G}$ is an ability to support a relatively high data rate (targets at $1 \mathrm{Gbit} / \mathrm{s}$ ) through the downlink [1-5]. There is already a simmering interest in the technologies beyond 4G, and the industry and academia are launching new projects with the focus on such technologies. However, the industry's view of the future wireless standard is mostly focused on data rates and efficiency. A 5G approach must be able to efficiently support different traffic types, which all have to be part of future wireless cellular systems. The vision of a unified frame structure concept, depicted by several authors, and authors' main aims are to handle a large set of requirements in a single 5G system. The 5G of wireless/mobile broadband technology has the properties of numerous devices support, network interconnection and high traffic throughput [2-6]. The heterogeneity is a feature that is expected to characterize the emerging wireless world, as a mixture of cells of diverse sizes and access points with different characteristics and technologies is used. Intelligence can be provided in terms of energy and cost-efficient solutions, according to which a certain application/service/quality provision is achieved. In particular, the introduction of intelligence in heterogeneous network deployments and the cloud radio-access network (RAN) is investigated in [1]. The main attributes of 5G technologies have the following contributory areas: Massive MIMO, Device Centric Communication, Smarter Device-toSmarter Device, Native support for Machine-to-Machine Communications and Millimetre wave 
communications etc. $[2,4,7,8,9,11,12]$. The Massive MIMO of the key point in 5G networks has been made to achieve the spectral efficiency, which was not possible without the increased BS densification. The smoothed out channel responses were mandatory, because of the vast spatial diversity, which brings about the favourable action of the law of large numbers. In this paper, we focus on a device centric communication to support the $5 \mathrm{G}$ networks, which has been derived from $\mathrm{D} 2 \mathrm{D}$ communications. Here, we are addressing a device centric feature of $5 \mathrm{G}$ networks. There are some technologies, such as Wi-Fi or Bluetooth, used to provide some D2D communication functionality. However, they work based on the unlicensed band, and their interference is uncontrollable. In addition, they cannot provide security and quality of service (QoS) guarantee for PCS networks. The D2D working group does not want to lose the emerging D2D market, the cellular operators and vendors, who are exploring the possibilities of introducing a D2D communication capability into cellular networks, which has sparkled an interest in this topic. Some recent works on D2D in cellular systems have reported results on interference management issues and radio resource allocation as well as on communication session setup and management procedures [10-13].

The peak rate is the best-case data rate that a user can hope to achieve under any conceivable network configuration. To achieve these targets, we propose a mechanism for D2D communication session setup and management procedures in the cellular system architecture and we present numerical results based on system simulations in an interference limited local area scenario. Our results show that D2D communication can increase the total throughput over the traditional techniques.

\section{The Proposed Work}

In this section, we introduce a new technique for the D2D communication system over the $5 \mathrm{G}$ networks. In order to support higher throughput, a $5 \mathrm{G}$ cellular networks working group must achieve less latencies, lower energy consumption, lower costs, and support many low-rate connections. The low energy consumption and cost can be dealt with at less cost. In this section, we discuss important ongoing research areas that support these requirements. The fundamental aspects of the $5 \mathrm{G}$ physical layer are: the waveform, evolution of the cloud-based system with virtualized network architectures, latency and control signalling, and energy efficiency.

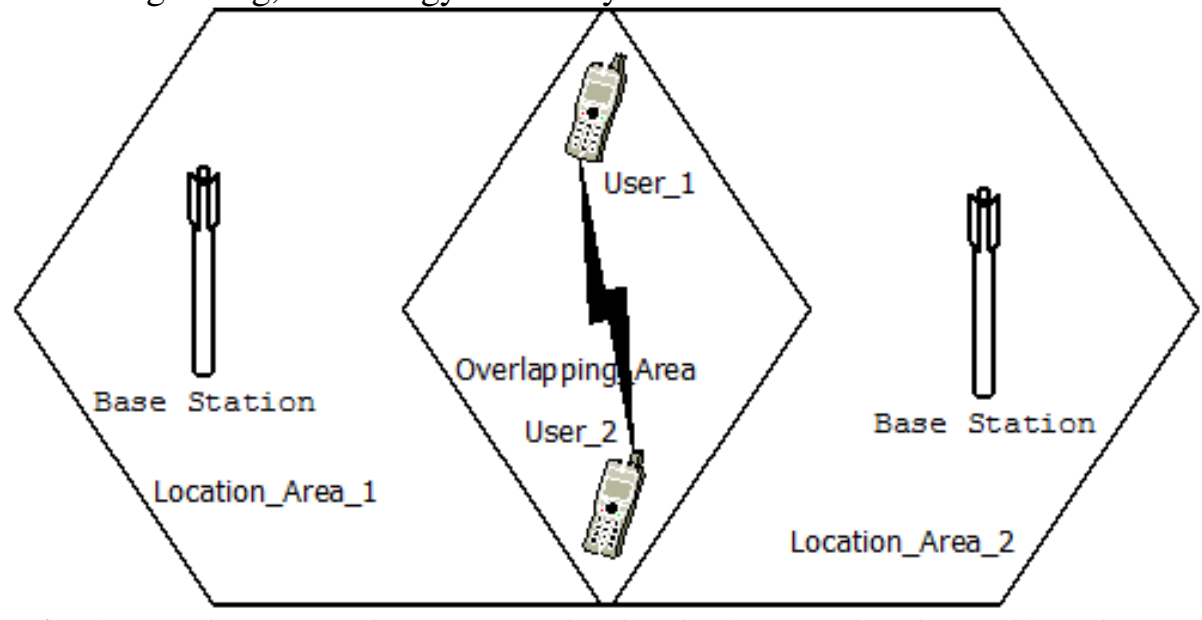

Figure 1. The Device-to-Device Communication in the Overlapping cell: A 5G Approach

In order to overcome initiative issues in cellular communications, we are proposing a new technique for device centric communication in 5G networks, and it has the following steps (as shown in Figure 1).

- The User Equipment (UE) initiates the call.

- The associated BS is used to start the communication and put an effort to the request packet to Base Station Controller (BSC) or Mobile Switching Centre (MSC). The MSC/BSC used to setup a call between the UE and the Corresponding Node $(\mathrm{CN})$.

- The UE starts the communication with the help of the BS. 
- The network computes the position of the UE and CN.

- If the UE and CN are in the neighbouring cell or the overlapping area, MSC will transfer the call to a D2D communication system. In most cases, they receive the signals from multiple BS. In contemporary approach cells are very small and they have a radius of few meters like LTE.

- This handover will take place with respect to the signal strength. It can be concluded that the proposed work will reduce the signalling overheads, cost and also lead to green communications.

\section{Performance Analysis}

In this section, we will compute the performance of the proposed work. It has been observed that the proposed scheme has less signalling cost. Here, we compare our scheme with the traditional scheme of cellular communication such as $4 \mathrm{G}$ and LTE schemes.

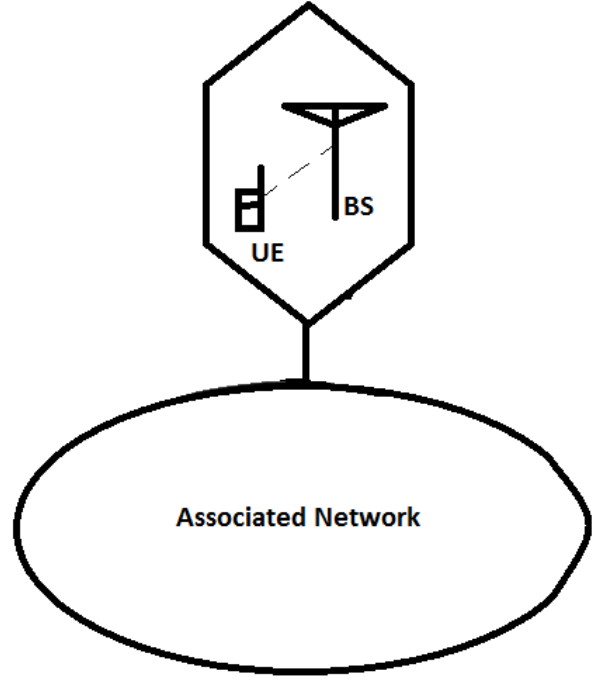

Inter-Cell Movement

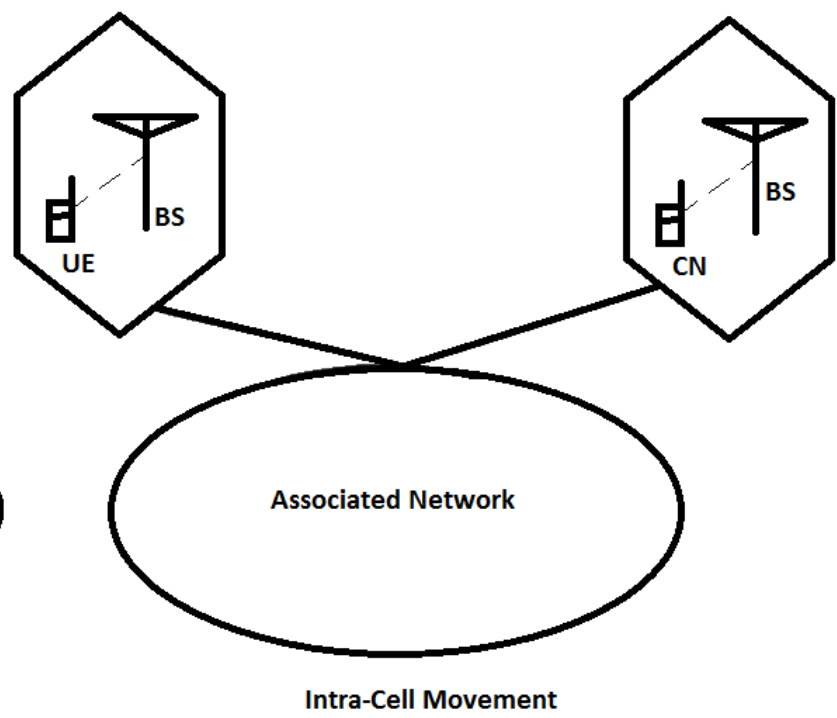

Intra-Cell Movement

Figure 2. The User Movements

The performance analysis of the proposed work has been computed by some numerical methods. The mathematical symbols are listed in Table 1. The signalling cost depends on several factors and but is not limited to hops counts $(h)$, bandwidth consumption and the number of active users per location area. In this paper, we consider the performance of the proposed work on the basis of the above mentioned factors. The user movement can be classified into inter cell movements and intra-cell movements, as shown in Figure 2.

Intra-cell: The total signalling cost can be classified as UE $\rightarrow \mathrm{BS}$, $\mathrm{BS} \rightarrow \mathrm{MSC} / \mathrm{BTS}$, MSC $\rightarrow \mathrm{MSC}$, $\mathrm{MSC} \rightarrow \mathrm{T}-\mathrm{BS}, \mathrm{BS} \rightarrow \mathrm{CN}$. Inter-cell: The total signalling cost can be classified as UE $\rightarrow$ BS, BS $\rightarrow$ MSC, $\mathrm{MSC} \rightarrow \mathrm{T}-\mathrm{BS}, \mathrm{BS} \rightarrow \mathrm{CN}$.

The signalling cost can be computed as the proposed technique, and it can be calculated with every threshold distance " $d$ " for the overlapping signalling strength. The signalling cost depends on three components: signalling cost to travel to the signal, binding cost for data packets, and packets delivery cost. The signalling cost for travel incurs with the transmission cost and processing cost based on per unit of distance $(d)$, and it is proportional to the number of UE. Then, we have $C_{T}^{d}=N_{U E} \frac{T_{C}+T_{P}}{d}$.

Table 1: Symbols and Their Description 


\begin{tabular}{ll}
\hline Symbols & Description \\
\hline$\eta$ & Proportionality constant \\
\hline$v$ & Association lookup cost per unit \\
\hline$\chi$ & Liner coefficient of location management(LM) \\
\hline$\lambda_{\mathrm{CALL}} / \lambda_{\text {cell }}$ & $\begin{array}{l}\text { An average number of calls to a target mobile terminal (MT) per unit } \\
\text { time in a LA/cell (Poission distribution random variable rate) }\end{array}$ \\
\hline$T_{C} / T_{P}$ & Transmission cost/ Processing Cost \\
\hline $\mathrm{SR}_{\mathrm{RREQ}} / \mathrm{SR}_{\mathrm{RREP}}$ & The size of the route request and the reply message for location update \\
\hline$\varpi / \phi$ & (LU) \\
\hline $\mathrm{d} / \mathrm{S}$ & Transmission cost per hop LU/Binding \\
\hline$\delta$ & Distance threshold/ An area of the cell \\
\hline $\mathrm{N}_{\mathrm{UE}}$ & Lookup cost per location database \\
\hline $\mathrm{B}_{\mathrm{UC}} / \mathrm{D}_{\mathrm{p}}$ & The number of user's equipment (a mobile user) within the location area \\
\hline
\end{tabular}

It is observed that all MSCs are interlinked with the wired medium and they will follow the medium access control (MAC) protocol. Therefore, the proportionality constant, the number of hops per hop transmission cost is interdependent when computing $T_{C}$. Thus, $T_{C}=2(h-1+\eta) \varpi$, and we have $\mathrm{C}_{\mathrm{T}}^{\mathrm{d}}=\mathrm{N}_{\mathrm{UE}} \times \frac{2(\mathrm{~h}-1+\eta) \mathrm{v}+\mathrm{T}_{\mathrm{P}}}{\mathrm{d}}$. Similarly, we can calculate the binding update cost: $\mathrm{C}_{\mathrm{BU}-\mathrm{T}}^{\mathrm{d}}=\mathrm{N}_{\mathrm{UE}} \times \mathrm{N}_{\mathrm{CN}} \times \frac{\mathrm{B}_{\mathrm{UC}}}{\mathrm{d}}$, where $\mathrm{B}_{\mathrm{UC}}=2(\mathrm{~h}-1+\eta) \varphi$. Here, $\mathrm{C}_{\mathrm{BU}-\mathrm{T}}^{\mathrm{d}}=\mathrm{N}_{\mathrm{UE}} \times \mathrm{N}_{\mathrm{CN}} \times \frac{2(\mathrm{~h}-1+\eta) \varphi}{\mathrm{d}}$.

The packet delivery cost can be computed as $\mathrm{C}_{\mathrm{PD}-\mathrm{T}}^{\mathrm{d}}=\mathrm{N}_{\mathrm{UE}} \times \mathrm{N}_{\mathrm{CN}} \times v[14]$ where, $v=\frac{\delta \lambda_{\mathrm{CALL}}}{\mathrm{S}}=\frac{\chi \mathrm{N}_{\mathrm{UE}} \lambda_{\mathrm{CALL}}}{\mathrm{S}}$, where $\delta=\chi \cdot \mathrm{N}_{\mathrm{UE}} \cdot$ So, $\mathrm{C}_{\mathrm{PD}-\mathrm{T}}^{\mathrm{d}}=\mathrm{N}_{\mathrm{UE}}^{2} \times \mathrm{N}_{\mathrm{CN}} \times \frac{\chi \lambda_{\mathrm{CALL}}}{\mathrm{S}}$.

Then, the per unit signalling cost $\left(\mathrm{C}_{\text {Signal }}\right)$ can be computed as:

$\mathrm{C}_{\text {Signal }}=\left(\mathrm{C}_{\mathrm{T}}^{\mathrm{d}}+\mathrm{C}_{\mathrm{BU}-\mathrm{T}}^{\mathrm{d}}+\mathrm{C}_{\mathrm{PD}-\mathrm{T}}^{\mathrm{d}}\right) \times\left(\mathrm{SR}_{\mathrm{RREQ}}+\mathrm{SR}_{\mathrm{RREP}}\right)$

$\mathrm{C}_{\text {signal }}=\left(\mathrm{N}_{\mathrm{MT}} \times \frac{2(\mathrm{~h}-1+\eta) \mathrm{v}+\mathrm{T}_{\mathrm{P}}}{\mathrm{d}}+\mathrm{N}_{\mathrm{UE}} \times \mathrm{N}_{\mathrm{CN}} \times \frac{2(\mathrm{~h}-1+\eta) \phi}{\mathrm{d}}+\mathrm{N}_{\mathrm{UE}}^{2} \times \mathrm{N}_{\mathrm{CN}} \times \frac{\chi \lambda_{\mathrm{CALL}}}{\mathrm{S}}\right) \times\left(\mathrm{SR}_{\mathrm{RREQ}}+\mathrm{SR}_{\mathrm{RREP}}\right)$.

Here, we assume that the intra-cell and the inter-cell have at least 4 and 5 units of signalling costs, respectively [14]. Now, we are taking the average of the duration of transmission of data packets to be equal to $t$. So, the signalling cost of the network can be computed as

$\mathrm{C}_{\text {Signal }}^{\text {Tradional }}=\left(\mathrm{N}_{\mathrm{MT}} \times \frac{2(\mathrm{~h}-1+\eta) \mathrm{v}+\mathrm{T}_{\mathrm{P}}}{\mathrm{d}}+\mathrm{N}_{\mathrm{UE}} \times \mathrm{N}_{\mathrm{CN}} \times \frac{2(\mathrm{~h}-1+\eta) \phi}{\mathrm{d}}+\mathrm{N}_{\mathrm{UE}}^{2} \times \mathrm{N}_{\mathrm{CN}} \times \frac{\chi \lambda_{\mathrm{CALL}}}{\mathrm{S}}\right) \times\left(\mathrm{SR}_{\mathrm{RREQ}}+\mathrm{SR}_{\mathrm{RREP}}\right) \times\left(D_{p} \times t\right)$

In our proposed scheme, the first data packet will follow the same path as the traditional scheme will. Later, it sets up a D2D communication path, so we can compute the same equation as follows. Let us note that when $\mathrm{N}_{\mathrm{UE}}=\mathrm{N}_{\mathrm{CN}}=1$, it shows a one-to-one communication link. 


$$
\begin{aligned}
& \mathrm{C}_{\text {Proposed }}=\left(\mathrm{N}_{\mathrm{UE}} \times \frac{2(\mathrm{~h}-1+\eta) \mathrm{v}+\mathrm{T}_{\mathrm{P}}}{\mathrm{d}}+\mathrm{N}_{\mathrm{UE}} \times \mathrm{N}_{\mathrm{CN}} \times \frac{2(\mathrm{~h}-1+\eta) \phi}{\mathrm{d}}+\mathrm{N}_{\mathrm{UE}}^{2} \times \mathrm{N}_{\mathrm{CN}} \times \frac{\chi \lambda_{\mathrm{CALL}}}{\mathrm{S}}\right) \\
& \times\left(\mathrm{SR}_{\mathrm{RREQ}}+\mathrm{SR}_{\mathrm{RREP}}\right) \times\left(D_{p}\right)+\left(\frac{2(\mathrm{~h}-1+\eta) \mathrm{v}+\mathrm{T}_{\mathrm{P}}}{\mathrm{d}}+\frac{2(\mathrm{~h}-1+\eta) \phi}{\mathrm{d}}+\frac{\chi \lambda_{\mathrm{CALL}}}{\mathrm{S}}\right) \times\left(\mathrm{SR}_{\mathrm{RREQ}}+\mathrm{SR}_{\mathrm{RREP}}\right) \times\left(D_{p} \times(t-1)\right)
\end{aligned}
$$

\section{Numerical Results}

Now we will check the efficiency and effectiveness of the proposed work in simulations. The initial parameters set is as follows. There are 5 users in the cell and they communicate to $\mathrm{CN}$. The average hop distance between the caller and called users is 5 . The minimum threshold value of handover is 50 meters. The transition probability constant is 0.02 , the size of the route request and the route reply packet size is 256 bytes, and size of the data packet has also the same size. The initial transmission and processing cost is assumed to be 0.02 units. The area of the each cell is $500 \mathrm{~m}^{2}$.

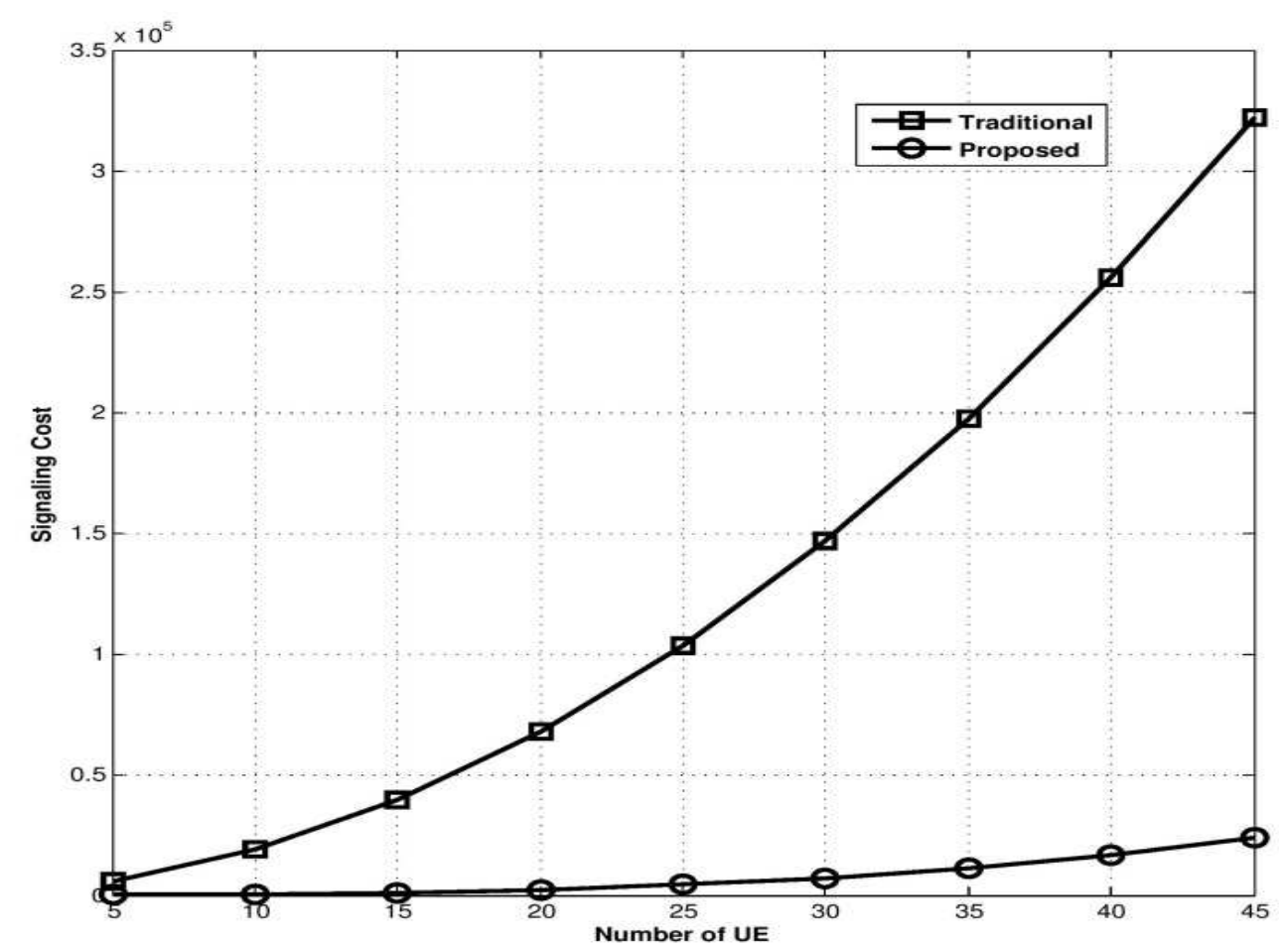

Figure 3: Signalling Cost Vs Number of UE in a cell

Figure 3 shows the effect of the number of mobile users in a cell and it varies the communication in corresponding nodes. Here, the number of UE is varying from 5 to 45 , and the same refers to the number of CNs. It has been observed that when the number of users is increasing, the overall cost will be increased also, but in the proposed work, the signal cost does not increase as compare to the traditional approach. As the first packet will be routed via the BS, the rest of the data packet will move through the D2D system.

\section{Conclusions}

In this paper, we have proposed a device centric communication system for the $5 \mathrm{G}$ communication architecture. When a mobile user receives the multiple signals for multiple cells, then the user should be handed over to an adjacent or neighbouring cell, and this process leads to signalling overheads. To overcome this issue, we proposed to start the device centric communication in the overlapping area. The initial phase of the communication is network centric and after that it should be handed over to a user centric mode. The performance analysis of the proposed work shows the effectiveness of the 
work, and a significant improvement in comparison to the traditional system has been observed in terms of signalling cost.

\section{Acknowledgments}

This work is funded (in part) by the Russian Federal budget funds for research work (Fundamental research, applied research and experimental development), grant No. 3942, and performed in accordance with Russian Government Resolutions No. 2014/226 of 2016, and by the Postdoctoral Grant of National Research Tomsk Polytechnic University, Russia.

\section{References}

[1] Qian Li, Huaning Niu, Apostolos Papathanassiou, Geng Wu 2014 5G Network Capacity Key Elements and Technologies. IEEE Vehicular Technology Magazine. 9(1) 71- 78

[2] Theodore. S. Rappaport, Shu Sun, Imma Mayzus, Hang Zhao, Yaniv Azar, Kevin Wang, George n. Wong, Jocelyn k. Schulz, Mathew Samimi, Felix Gutierrez 2013 Millimeter Wave Mobile Communications for 5G Cellular: It Will Work!. IEEE Access. 1(1) 335 - 349

[3] Ian F. Akyildiz, David M. Gutierrez-Estevez, Elias Chavarria Reyes 2010 The evolution to 4G cellular systems: LTE-Advanced. Physical Communication 3(4) 217-244

[4] Jeffrey G. Andrews, Stefano Buzzi, Wan Choi, Stephen V. Hanly, Angel Lozano, Anthony C. K. Soong, Jianzhong Charlie Zhang 2014 What Will 5G Be. IEEE Journal On Selected Areas In Communication. 32(6) 1065-1082

[5] Federico Boccardi, Robert W. Heath Jr., Angel Lozano, Thomas L. Marzetta, Petar Popovski 2014 Five disruptive technology directions for 5G. IEEE Communications Magazine. 52(2) 7480

[6] Daquan Feng, Lu Lu, Yi Yuan-Wu, Geoffrey Ye Li, Gang Feng, and Shaoqian Li 2013 Deviceto-Device Communications Underlaying Cellular Networks. IEEE Transactions On Communications. 61(8) 3541-3551

[7] Gerhard Fettweis, Siavash Alamouti 2014 5G: Personal Mobile Internet beyond What Cellular Did to Telephony. IEEE Communications Magazine. 52(2) 140-145

[8] Volker Jungnickel, Konstantinos Manolakis, Wolfgang Zirwas, Berthold Panzner, Volker Braun, Moritz Lossow, Mikael Sternad, Rikke Apelfröjd, Tommy Svensson 2014 The role of small cells, coordinated multipoint, and massive MIMO in 5G. IEEE Communications Magazine. 52(2) $44-51$

[9] Lei Lei, Zhangdui Zhong, Chuang Lin, Xuemin Shen 2012 Operator Controlled Device -ToDevice Communications in LTE Advance Networks. IEEE Wireless Communications. 19(3) $96-104$

[10] Josef Noll, Mohammad M. R. Chowdhury 2011 5G: Service Continuity in Heterogeneous Environments. Wireless Personal Communications. 57(3) 413-429

[11] Afif Osseiran, Federico Boccardi, Volker Braun, Katsutoshi Kusume, Patrick Marsch, Michal Maternia, Olav Queseth, Malte Schellmann, Hans Schotten, Hidekazu Taoka, Hugo Tullberg, Mikko A. Uusitalo, Bogdan Timus, Mikael Fallgren 2014 Scenarios for 5G Mobile and Wireless Communications: The Vision of the METIS Project. IEEE Communications Magazine. 52(5) 26- 35

[12] Tomislav Shuminoski, Toni Janevski 2014 Radio Network Aggregation for 5G Mobile Terminals in Heterogeneous Wireless and Mobile Networks. Wireless Personal Communication. 78(2) 1211-1229

[13] Mohsen Nader Tehrani, Murat Uysal, and Halim Yanikomeroglu 2014 Device-to-Device Communication in 5G Cellular Networks: Challenges, Solutions, and Future Directions. IEEE Communications Magazine.52(5) 86-92

[14] Sanjay Kumar Biswash, Chiranjeev Kumar 2011 An efficient metric-based (EM-B) location management scheme for wireless cellular networks. Journal of Network and Computer Applications. 34(6) 2011-2026 